\title{
ON A VARIATIONAL APPROXIMATION METHOD FOR A CLASS OF ELLIPTIC EIGENVALUE PROBLEMS IN COMPOSITE STRUCTURES
}

\author{
M. VANMAELE AND R. VAN KEER
}

\begin{abstract}
We consider a second-order elliptic eigenvalue problem on a convex polygonal domain, divided in $M$ nonoverlapping subdomains. The conormal derivative of the unknown function is continuous on the interfaces, while the function itself is discontinuous. We present a general finite element method to obtain a numerical solution of the eigenvalue problem, starting from a nonstandard formally equivalent variational formulation in an abstract setting in product Hilbert spaces. We use standard Lagrange finite element spaces on the subdomains. Moreover, the bilinear forms are approximated by suitable numerical quadrature formulas. We obtain error estimates for both the eigenfunctions and the eigenvalues, allowing for the case of multiple exact eigenvalues, by a pure variational method.
\end{abstract}

\section{INTRODUCTION}

Let $\Omega \subset \mathbb{R}^{2}$ be a bounded convex polygonal domain with boundary $\partial \Omega$. Assume that $\partial \Omega=\Gamma_{1} \cup \Gamma_{2} \cup \Lambda_{1}$, where $\Gamma_{1}$ and $\Gamma_{2}$ are open and consist of an integer number of sides, $\Gamma_{1} \cap \Gamma_{2}=\emptyset$ and meas $\Lambda_{1}=0$. Further, let $\Omega$ be divided in $M$ nonoverlapping, open, convex, polygonal domains $\Omega_{i} \subset \Omega, 1 \leq i \leq M$. We denote by $\mathcal{N}_{i} \subset\{1, \ldots, M\}$ the set of integers $\sigma$ for which meas $1\left(\partial \Omega_{i} \cap \partial \Omega_{\sigma}\right)>0$. We set $\Gamma_{i, \sigma}=\partial \Omega_{i} \cap \partial \Omega_{\sigma}$ for $\sigma \in \mathcal{N}_{i}, 1 \leq i \leq M$. We assume that $\Gamma_{i, \sigma} \cap \Gamma_{j, \rho}=\emptyset$ when $\{i, \sigma\} \neq\{j, \rho\}$.

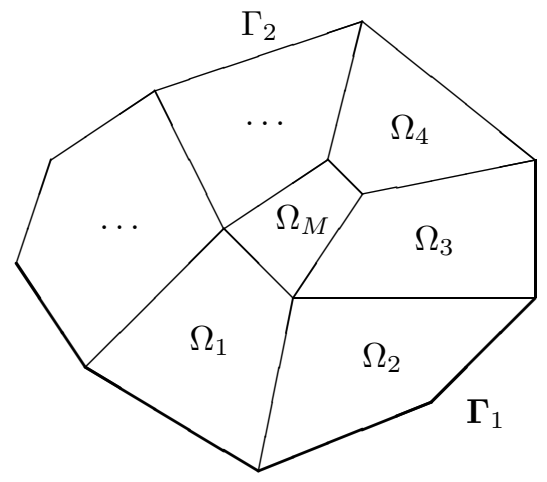

Received by the editor August 23, 1993 and, in revised form, January 23, 1995

1991 Mathematics Subject Classification. Primary 65N25, 65N30, 65D30.

(C)1996 American Mathematical Society 
Then we may write

$$
\Omega=\bigcup_{i=1}^{M} \Omega_{i} \bigcup_{i=1}^{M}\left(\bigcup_{\substack{\sigma \in \mathcal{N}_{i} \\ \sigma>i}} \Gamma_{i, \sigma}\right) \cup \Lambda_{2}, \quad \operatorname{meas}_{1} \Lambda_{2}=0 .
$$

Finally, let $\mathcal{N}\left(\Gamma_{j}\right)=\left\{i: 1 \leq i \leq M\right.$ and $\left.\operatorname{meas}_{1}\left(\Gamma_{j} \cap \partial \Omega_{i}\right)>0\right\}, j=1,2$, and $\Gamma_{j}^{i}=\Gamma_{j} \cap \partial \Omega_{i}, i \in \mathcal{N}\left(\Gamma_{j}\right), j=1,2$. In what follows, when writing $\Gamma_{j}^{i}$, we will sometimes delete the restriction $i \in \mathcal{N}\left(\Gamma_{j}\right)$, of course taking $\Gamma_{j}^{i}=\emptyset$ when $i \notin \mathcal{N}\left(\Gamma_{j}\right)$.

This paper deals with the eigenvalue problem (EVP) of determining the real numbers $\lambda$ and the corresponding functions $u^{i}: \Omega_{i} \rightarrow \mathbb{R}, 1 \leq i \leq M$, that obey, in a weak sense, the differential equation

$$
-\sum_{\ell, m=1}^{2} \frac{\partial}{x_{\ell}}\left(a_{\ell m}^{i} \frac{\partial u^{i}}{\partial x_{m}}\right)+a_{0}^{i} u^{i}=\lambda u^{i} \quad \text { in } \Omega_{i},
$$

together with the following transmission conditions (TCs):

$$
\begin{aligned}
& -a^{i} \partial_{\nu} u^{i}=h^{i, \sigma}\left(u^{i}-u^{\sigma}\right) \quad \text { on } \Gamma_{i, \sigma}, \\
& \forall \sigma \in \mathcal{N}_{i} \\
& a^{i} \partial_{\nu} u^{i}=a^{\sigma} \partial_{\nu} u^{\sigma} \quad \text { on } \Gamma_{i, \sigma},
\end{aligned}
$$

as well as with the homogeneous boundary conditions (BCs)

$$
\begin{array}{rlrl}
u^{i} & =0 & & \text { on } \Gamma_{1}^{i}, \\
-a^{i} \partial_{\nu} u^{i}+a_{1}^{i} u^{i}=0 & & \text { on } \Gamma_{2}^{i} .
\end{array}
$$

The conormal derivative in (1.5) is given by

$$
a^{i} \partial_{\nu} u^{i}=\sum_{\ell, m=1}^{2} a_{\ell m}^{i} \frac{\partial u^{i}}{\partial x_{m}} \nu_{\ell},
$$

with $\nu_{\ell}$ the $\ell$ th component of the unit outer normal vector $\bar{\nu}$ to $\Gamma_{2}^{i}$. The conditions (1.2)-(1.3) have to be understood similarly, $\bar{\nu}$ being the unit normal vector to $\Gamma_{i, \sigma}$ and pointing from $\Omega_{i}$ to $\Omega_{\sigma}$. In this problem $a_{\ell m}^{i}, a_{0}^{i}, h^{i, \sigma}$ and $a_{1}^{i}, 1 \leq \ell, m \leq 2$, $\sigma \in \mathcal{N}_{i}, 1 \leq i \leq M$, are given space-dependent functions, which are sufficiently regular, as specified below.

In fact, it is precisely (1.2) which makes the present EVP a nonstandard one. In the usual transition (eigenvalue or boundary value) problems in composite media both the unknown and its conormal derivative are continuous at the interface of two subregions, in spite of a discontinuity of the coefficients in the differential equation at this internal boundary. Such problems have a well-known variational formulation, see e.g. [4, Chapitre VII, Section 2], and their finite element approximation offers no special difficulties. The meaning and origin of the TC (1.2) are different than for the usual continuity condition. Moreover, (1.2) prevents the variational approach for standard transition problems to be applicable.

We overcome this difficulty by passing to a product space setting of the present EVP, allowing us to derive a suitable but nonstandard formally equivalent variational formulation in an abstract setting, which explicitly reflects the TC (1.2). This variational form of the EVP serves as the starting point for a finite element approximation method (FEM), both with and without numerical quadrature, also 
in a product space setting. Moreover, when suitably identifying the canonical basis for the finite element product spaces, the method is found to be attractive from the computational point of view too, as the stiffness and mass matrices of the resulting algebraic EVPs can be constructed by means of the stiffness and mass matrices for one-component problems on the respective domains $\Omega_{i}, 1 \leq i \leq M$. This variational approach to the EVP (1.1)-(1.5) is thought to be new.

The TC (1.2) arises in a natural way in various practical applications, for instance in the context of transient heat transfer problems in composite media with nonperfect thermal contacts at the internal boundaries, see e.g. [11, Chapters 8], [10, Chapter 9] and [7]. Only in very special cases can the exact eigenpairs of the EVP (1.1)-(1.5) be obtained; cf. the first two references just quoted.

The strategy for the paper is as follows. We first show that the present type of EVPs indeed fits into the general framework of abstract EVPs for symmetric, coercive and bounded bilinear forms in Hilbert spaces. Hence, classical arguments imply the existence of exact eigenpairs, obeying some standard properties. By the properties of suitably chosen approximation spaces with triangular or, where possible, with rectangular elements and by the property of the properly introduced Lagrange interpolation and elliptic projection, the convergence and error estimates of the consistent mass FEM are similar to those for standard elliptic 2nd-order EVPs. Next, to incorporate the effect of a suitable numerical quadrature on the approximate eigenpairs, we proceed to some extent similarly as in $[1,15,14]$. Apart from the structure of $\Omega$ and of the corresponding product (approximation) function spaces, the main difficulty concerns the line integrals on the interfaces $\Gamma_{i, \sigma}$, arising from the TCs (1.2)-(1.3).

In the error analysis we allow for the case of multiple exact eigenvalues.

An outline of the paper is now in order. The precise variational formulation of the EVP (1.1)-(1.5), which is crucial for our approach, is stated in $\S 2$, together with some preliminary results concerning the function spaces and the bilinear form. This allows the EVP to be put in the framework mentioned above. In $\S 3$ we first introduce suitable approximation spaces and then the elliptic projector. The consistent mass FEM is dealt with in $\S 4$, while the numerical quadrature FEM is discussed in detail in $\S 5$. To illustrate the effectiveness of the present approach, we consider in $\S 6$ a 1D-analogue of the EVP (1.1)-(1.5), the exact eigenpairs of which are easily found. Finally, in the Appendix we give a physical example of the origin of the TCs (1.2)-(1.3).

\section{VARIATIONAL EIGENVALUE PROBLEM}

2.1. Notations and assumptions. Let $H^{1}\left(\Omega_{i}\right)$ be the usual first-order Sobolev space on $\Omega_{i}$ with norm $\|\cdot\|_{1, \Omega_{i}}, 1 \leq i \leq M$, and let $V^{i}=\left\{w \in H^{1}\left(\Omega_{i}\right): w=\right.$ 0 on $\left.\Gamma_{1}^{i}\right\}$. We recall that $\Gamma_{1}^{i}=\emptyset$ when $\operatorname{meas}_{1}\left(\Gamma_{1} \cap \partial \Omega_{i}\right)=0$. Then, we introduce the product space

$$
V=\left\{v=\left(v^{1}, \ldots, v^{M}\right): v^{i} \in V^{i}, 1 \leq i \leq M\right\}
$$

and we identify $v \in V$ with a scalar function $v: \Omega \rightarrow \mathbb{R}$ for which $\left.v\right|_{\Omega_{i}}=v^{i}$ on $\Omega_{i}$, $1 \leq i \leq M$. Similarly, we introduce the product space $H=L_{2}\left(\Omega_{1}\right) \times \cdots \times L_{2}\left(\Omega_{M}\right)$ 
with inner product $(\cdot, \cdot)$ and associated norm $|\cdot|$ given by

$$
(v, w)=\sum_{i=1}^{M} \int_{\Omega_{i}} v^{i} w^{i} \mathrm{~d} x, \quad|v|=\sqrt{(v, v)}, \quad \forall v, w \in H .
$$

Further, let

$$
\begin{aligned}
& \mathcal{A}(v, w)=\sum_{i=1}^{M} \int_{\Omega_{i}}\left(\sum_{\ell, m=1}^{2} a_{\ell m}^{i} \frac{\partial v^{i}}{\partial x_{\ell}} \frac{\partial w^{i}}{\partial x_{m}}+a_{0}^{i} v^{i} w^{i}\right) \mathrm{d} x, \\
& \mathcal{B}(v, w)=\frac{1}{2} \sum_{i=1}^{M} \sum_{\sigma \in \mathcal{N}_{i}} \int_{\Gamma_{i, \sigma}} h^{i, \sigma}\left(v^{i}-v^{\sigma}\right)\left(w^{i}-w^{\sigma}\right) \mathrm{d} s \\
& \mathcal{C}(v, w)=\sum_{i \in \mathcal{N}\left(\Gamma_{2}\right)} \int_{\Gamma_{2}^{i}} a_{1}^{i} v^{i} w^{i} \mathrm{~d} s
\end{aligned}
$$

and

$$
a(v, w)=\mathcal{A}(v, w)+\mathcal{B}(v, w)+\mathcal{C}(v, w), \quad \forall v, w \in V .
$$

We will also use the product space $\widehat{H}^{m}(\Omega)=H^{m}\left(\Omega_{1}\right) \times \cdots \times H^{m}\left(\Omega_{M}\right), m \in \mathbb{N}_{0}$, and its (product) norm $\|\cdot\|_{\widehat{H}^{m}(\Omega)}$ and (product) seminorm $|\cdot|_{\widehat{H}^{m}(\Omega)}$ both defined in the natural way. For $m=1$, the product norm is simply denoted by $\|\cdot\|$.

Throughout this paper, the data are assumed to fulfill the hypotheses (H1)-(H2):

1) $a_{\ell m}^{i}, a_{0}^{i} \in L_{\infty}\left(\Omega_{i}\right) ; a_{\ell m}^{i}=a_{m \ell}^{i}$ a.e. in $\Omega_{i}, \quad \ell, m=1,2,1 \leq i \leq M$;

(2) $h^{i, \sigma} \in L_{\infty}\left(\Gamma_{i, \sigma}\right) ; 0<h^{i, \sigma}=h^{\sigma, i}$ a.e. on $\Gamma_{i, \sigma}, \quad \sigma \in \mathcal{N}_{i}, 1 \leq i \leq M$;

(3) $a_{1}^{i} \in L_{\infty}\left(\Gamma_{2}^{i}\right) ; \exists \alpha_{1} \geq 0: a_{1}^{i} \geq \alpha_{1}$ a.e. on $\Gamma_{2}^{i}, \quad i \in \mathcal{N}\left(\Gamma_{2}\right)$.

(H2) (1) The matrices $a^{i}=\left(a_{\ell m}^{i}\right), 1 \leq i \leq M$, are positive definite, i.e.,

$\exists \alpha>0: \forall \xi \in \mathbb{R}^{2}, \sum_{l, m=1}^{2} a_{l m}^{i}(x) \xi_{l} \xi_{m} \geq \alpha|\xi|^{2}$ a.e. in $\Omega_{i}, \quad 1 \leq i \leq M ;$

(2) $\exists \alpha_{0}>0: a_{0}^{i} \geq \alpha_{0}$ a.e. in $\Omega_{i}, \quad 1 \leq i \leq M$.

These hypotheses ensure the ellipticity of the EVP.

2.2. Variational formulation. The weak or variational EVP associated with (1.1)-(1.5) reads:

$$
\text { Find }(\lambda, u) \in \mathbb{R} \times V, u \neq 0: a(u, v)=\lambda(u, v), \quad \forall v \in V,
$$

where $(\cdot, \cdot)$ and $a(\cdot, \cdot)$ are defined by $(2.1)$ and $(2.5)$, respectively.

The integral identity (2.6) is obtained from (1.1)-(1.5) by first dealing with the EVP for the pair $\left(\lambda, u^{i}\right)$ in the usual way and by next summing up the resulting variational equation for $i=1, \ldots, M$; we use the symmetry $h^{i, \sigma}=h^{\sigma, i}$ and the notations (2.1)-(2.5).

The formal equivalence of the classical and the variational EVP may be shown by adapting standard arguments to the present product space setting. Particularly, to 
recover the TC (1.2) from (2.6), we use a well-known density property in $L_{2}\left(\Gamma_{i, \sigma}\right)$, see e.g. [9, Theorem 6.6.3].

To put (2.6) into a general framework of abstract variational elliptic EVPs in Hilbert spaces (see e.g. [13]), we rely on the following preliminary result, the proof of which is evident from the definitions of $H, V$ and $a(\cdot, \cdot)$.

Proposition 2.1. (1) The spaces $H$ and $V$ are Hilbert spaces, $V$ being compactly and densely embedded in $H$.

(2) The bilinear form $a(\cdot, \cdot): V \times V \rightarrow \mathbb{R},(2.5)$, is symmetric, bounded and strongly coercive.

From the classical theory of abstract elliptic EVPs we may now infer:

Theorem 2.1. (1) The EVP (2.6) has a countable infinite set of eigenvalues, all having finite multiplicity and being strictly positive, without finite accumulation point. We arrange them as

$$
0<\lambda_{1} \leq \lambda_{2} \leq \cdots \leq \lambda_{\ell} \leq \cdots \rightarrow \infty .
$$

(Here each eigenvalue occurs as many times as given by its multiplicity.)

(2) There is a Hilbert basis of $H$ formed by orthonormal eigenfunctions $u_{\ell}, \ell \geq$ 1. Furthermore, the sequence $\left(\lambda_{\ell}^{-1 / 2} u_{\ell}\right)_{\ell \geq 1}$ forms an orthonormal basis of $V$ with respect to $a(\cdot, \cdot)$.

We will deal with the approximation of the eigenpairs of (2.6).

\section{Preliminary Results}

3.1. Choice of approximation space. We consider a regular family of triangulations $\left(\mathcal{T}_{h_{i}}^{i}\right)$ (see e.g. [3]) of each component $\bar{\Omega}_{i}, 1 \leq i \leq M$, consisting of either triangular or, when $\Omega_{i}$ is a rectangle, rectangular elements with diameter $\leq h_{i}$. With a triangulation $\mathcal{T}_{h_{i}}^{i}$, we associate $X_{h_{i}}^{i}=\left\{v^{i} \in \mathcal{C}^{0}\left(\bar{\Omega}_{i}\right):\left.v^{i}\right|_{K} \in P(K), \forall K \in\right.$ $\left.\mathcal{T}_{h_{i}}^{i}\right\} \subset H^{1}\left(\Omega_{i}\right)$, where $h_{i}$ is the mesh parameter,

$$
P(K)=\left\{\begin{array}{ll}
P_{k}(K) & \text { if } K \text { is triangular, } \\
Q_{k}(K) & \text { if } K \text { is rectangular, }
\end{array} \quad k \in \mathbb{N},\right.
$$

and $P_{k}$ stands for the set of polynomials of degree $\leq k$ and $Q_{k}$ for the set of polynomials of degree $\leq k$ with respect to each variable. Next, let $V_{h_{i}}^{i}=\left\{v^{i} \in\right.$ $X_{h_{i}}^{i}: v^{i}=0$ on $\left.\Gamma_{1}^{i}\right\} \subset V^{i}$. Finally, we consider the product spaces

$$
\begin{aligned}
X_{h} & =\left\{v=\left(v^{1}, \ldots, v^{M}\right): v^{i} \in X_{h_{i}}^{i}, 1 \leq i \leq M\right\} \subset \widehat{H}^{1}(\Omega), \\
V_{h} & =\left\{v \in X_{h}: v^{i}=0 \text { on } \Gamma_{1}^{i}, 1 \leq i \leq M\right\} \subset V,
\end{aligned}
$$

where $h=\max _{1 \leq i \leq M} h_{i}$ is the overall mesh parameter, which we assume is sufficiently small.

In what follows, $C$ denotes a generic constant not depending on $h$ (even when it is not explicitly stated).

3.2. Inverse inequality. From now on, we assume that each regular family of triangulations $\left(\mathcal{T}_{h_{i}}^{i}\right), 1 \leq i \leq M$, is 'quasi-uniform' in the sense of [3, (3.2.28)]. Then, Theorem 3.2.6 in [3] implies that for $m \in \mathbb{N}_{0}$,

$$
|w|_{m, K} \leq C h_{i}^{-s}|w|_{m-s, K}, \quad 0 \leq s \leq m, \forall w \in P(K), \forall K \in \mathcal{T}_{h_{i}}^{i}, \forall h_{i},
$$




$$
\|w\|_{m, K} \leq C h_{i}^{-s}\|w\|_{m-s, K}, \quad 0 \leq s \leq m, \forall w \in P(K), \forall K \in \mathcal{T}_{h_{i}}^{i}, \forall h_{i} .
$$

Further, we assume that the families $\left(\mathcal{T}_{h_{i}}^{i}\right)_{h_{i}}$ are mutually 'quasi-uniform' too, i.e.,

$$
\exists \nu \geq 0: \frac{h}{h_{i}} \leq \nu \quad, \quad \forall h_{i}, 1 \leq i \leq M
$$

\subsection{Density and approximation property.}

Proposition 3.1. (1) The space $\widehat{H}^{2}(\Omega) \cap V$ is dense in $V$.

(2) The finite element space $V_{h} \subset V$ satisfies the approximation property

$$
\begin{aligned}
\inf _{v_{h} \in V_{h}}\left\{\left|v-v_{h}\right|+h\left|v-v_{h}\right|_{\widehat{H}^{1}(\Omega)}\right\} & \leq C h^{r+1}\|v\|_{\widehat{H}^{r+1}(\Omega)}, \\
\forall v & \in \widehat{H}^{r+1}(\Omega) \cap V, 1 \leq r \leq k .
\end{aligned}
$$

Proof. (1) Use the density of $H^{2}\left(\Omega_{i}\right) \cap V^{i}$ in $V^{i}, 1 \leq i \leq M$.

(2) Apply the approximation property of $V_{h_{i}}^{i}$ in $V^{i}, 1 \leq i \leq M$; see e.g. [13, p. 152] for a triangular mesh and e.g. [1, §5] for its direct analogue on a rectangular mesh.

3.4. The elliptic projector. We define the elliptic projector $P: V \rightarrow V_{h}$ by

$$
a(v-P v, w)=0, \quad \forall v \in V, \forall w \in V_{h} .
$$

From (3.4) one easily gets (cf. [13, Lemme 6.5-1] for a triangular mesh and [1, (6.2)] for a rectangular mesh)

$$
\|v-P v\| \leq C h^{k}\|v\|_{\widehat{H}^{k+1}(\Omega)}, \quad \forall v \in \widehat{H}^{k+1}(\Omega) \cap V .
$$

Similarly to [1, Lemma 6.1] (and its extension to triangular meshes) we get:

Proposition 3.2. Consider regular families of triangulations $\left(\mathcal{T}_{h_{i}}^{i}\right)_{h_{i}}$ of $\bar{\Omega}_{i}, 1 \leq$ $i \leq M$, satisfying the 'quasi-uniformity' property (3.3). Then we have

$$
\left(\sum_{i=1}^{M} \sum_{K \in \mathcal{T}_{h_{i}}^{i}}\left\|(P v)^{i}\right\|_{k+1, K}^{2}\right)^{1 / 2} \leq C\|v\|_{\widehat{H}^{k+1}(\Omega)}, \quad \forall v \in \widehat{H}^{k+1}(\Omega) \cap V .
$$

Remark 3.1. In the case of a triangular mesh on $\Omega_{i}$ one has $\|p\|_{k+1, K}=\|p\|_{k, K}$, for all $p \in P_{k}(K)$, for all $K \in \mathcal{T}_{h_{i}}^{i}$.

\section{Consistent mass finite Element method}

4.1. Variational eigenvalue problem. The consistent mass approximate EVP corresponding to (2.6) reads:

$$
\text { Find } \lambda_{h} \in \mathbb{R}, u_{h}=\left(u_{h}^{1}, \ldots, u_{h}^{M}\right) \in V_{h}: a\left(u_{h}, v\right)=\lambda_{h}\left(u_{h}, v\right), \quad \forall v \in V_{h} .
$$

The eigenpairs, the existence of which is guaranteed by the discrete analogue of Theorem 2.1, are denoted by $\left(\lambda_{\ell, h}, u_{\ell, h}\right), 1 \leq \ell \leq N(h)$.

From Proposition 3.1 a result analogous to Theorem 6.5-1 in [13] holds, viz. 
Theorem 4.1. (1) For $\ell=1, \ldots, N(h)$, we have

$$
0 \leq \lambda_{\ell, h}-\lambda_{\ell} \rightarrow 0 \quad \text { as } \quad h \rightarrow 0
$$

(2) Let $\lambda_{\ell}$ be a simple exact eigenvalue and $u_{\ell}$ the corresponding exact eigenfunction; then

$$
\left\|u_{\ell}-u_{\ell, h}\right\| \rightarrow 0 \quad \text { as } \quad h \rightarrow 0 .
$$

(3) Assume that the exact eigenfunctions $u_{j} \in \widehat{H}^{r+1}(\Omega), j=1, \ldots, \ell$. Then, for $1 \leq r \leq k$

$$
\lambda_{\ell, h}-\lambda_{\ell} \leq C\left(\lambda_{\ell}\right) h^{2 r}
$$

(4) Moreover, if $\lambda_{\ell}$ is a simple exact eigenvalue, then

$$
\left\|u_{\ell}-u_{\ell, h}\right\| \leq C\left(\lambda_{\ell}\right) h^{r} .
$$

Case of a multiple exact eigenvalue $\lambda_{\ell}$.

The assumption that $\lambda_{\ell}$ is simple was made in (4.3) and (4.5) only. This restriction may be dropped. Thus, let $\lambda_{\ell-1}<\lambda_{\ell}=\lambda_{\ell+1}=\cdots=\lambda_{\ell+L}<\lambda_{\ell+L+1}$. We denote the $L+1$ eigenfunctions associated with $\lambda_{\ell}$, chosen to be orthonormal in $H$, by $u_{\ell}, u_{\ell+1}, \ldots, u_{\ell+L}$. Further, let $\left(\lambda_{\ell+t, h}, u_{\ell+t, h}\right), t=0, \ldots, L$, be the corresponding eigenpairs of (4.1), the eigenfunctions being orthonormal in $H$.

Proceeding to some extent analogously as in $[5, \S$ XII.5.4], we readily obtain estimates similar to (4.5) for $U_{\ell+t, \star}(h)-u_{\ell+t, h}, 0 \leq t \leq L$, where $U_{\ell+t, \star}(h)$ are exact eigenfunctions corresponding to $\lambda_{\ell}$ and being orthonormal in $H$. Their dependence on $h$ is inconvenient for practical implementations. In the next theorem we overcome this difficulty.

Theorem 4.2. Let $1 \leq r \leq k$. Assume that $u_{j} \in \widehat{H}^{r+1}(\Omega), j=1, \ldots, \ell+L$. Then, there exist a set $\left(W_{\ell+t}\right)_{t=0}^{L}$ of fixed exact eigenfunctions corresponding to $\lambda_{\ell}$ that are orthonormal in $H$, a sequence $\left\{h_{j}\right\}$ with $h_{j} \rightarrow 0$ and a number $m, 0<m \leq k$, such that

$$
\left\|W_{\ell+t}-u_{\ell+t, h_{j}}\right\| \leq C(\ell) h_{j}^{m}, \quad t=0, \ldots, L .
$$

Proof. One first infers the existence of a set $\left(U_{\ell+t}(h)\right)_{t=0}^{L}$ of exact eigenfunctions of (2.6), corresponding to $\lambda_{\ell}$, such that

$$
\begin{aligned}
\left|U_{\ell+t}(h)-u_{\ell+t, h}\right| & \leq C(\ell) h^{k}, & & t=0, \ldots, L, \\
\left|\left(U_{\ell+t}(h), U_{\ell+s}(h)\right)\right| & \leq C(\ell) h^{k}, & & t \neq s, t, s=0, \ldots, L .
\end{aligned}
$$

The first estimate is shown similarly as in [5, (5.65), p.909], while the second estimate follows from (4.6) and

$$
\left(U_{\ell+t}(h), U_{\ell+s}(h)\right)=\left(U_{\ell+t}(h)-u_{\ell+t, h}, u_{\ell+s, h}\right)+\left(U_{\ell+t}(h), U_{\ell+s}(h)-u_{\ell+s, h}\right) .
$$

Next we slightly adapt the arguments in the second part of the proof of [16, Theorem 3.5] and those in the proof of [16, Theorem 3.7]. 
4.2. Algebraic eigenvalue problem. Choosing a basis of the product space $V_{h}$, we may rewrite (4.1) as an algebraic EVP in the standard way. We start from a usual Lagrange finite element basis $\left(\varphi_{r_{i}}^{i}\right)_{1 \leq r_{i} \leq I^{i}\left(h_{i}\right)}, I^{i}\left(h_{i}\right)=\operatorname{dim} X_{h_{i}}^{i}$, of $X_{h_{i}}^{i}, 1 \leq$ $i \leq M$. Here we take the underlying triangulations $\mathcal{T}_{h_{i}}^{i}$ and $\mathcal{T}_{h_{\sigma}}^{\sigma}$ of adjacent domains $\Omega_{i}$ and $\Omega_{\sigma}$ to share the nodes on the interface $\Gamma_{i, \sigma}$. Then the set $\left(\varphi_{s}\right)_{1 \leq s \leq I(h)}$, $I(h)=\operatorname{dim} X_{h}\left(=\sum_{i=1}^{M} I^{i}\left(h_{i}\right)\right)$, where

$$
\begin{aligned}
\varphi_{s} & =\left(\varphi_{s}^{1}, \ldots, \varphi_{s}^{M}\right), \\
\varphi_{s}^{i} & = \begin{cases}0 & \text { if the global node } s \text { does not belong to } \bar{\Omega}_{i}, \quad 1 \leq i \leq M, \\
\varphi_{r_{i}}^{i} & \text { if the global node } s \text { is the node } r_{i} \text { in } \bar{\Omega}_{i},\end{cases}
\end{aligned}
$$

forms a basis of the product space $X_{h}$. Deleting the basis functions corresponding to the nodes on $\Gamma_{1}$, we get a basis of $V_{h}$.

We emphasize that to a node on an interface $\Gamma_{i, \sigma}$, figuring both in $\mathcal{T}_{h_{i}}^{i}$ and $\mathcal{T}_{h_{\sigma}}^{\sigma}$, two basis functions are assigned.

As usual, all integrals entering the stiffness and mass matrices of the algebraic EVP may be reduced to integrals on a reference element $\widehat{K}$ and its sides.

Remark 4.1. The approximation method outlined above, from the variational formulation (2.6) to the choice of the canonical basis $V_{h}$ just made, is attractive from a computational point of view since one may start from existing codes for classical EVPs. Indeed, the stiffness and mass matrix of the resulting algebraic EVP show a block diagonal structure, the blocks originating from the stiffness and mass matrix of a scalar EVP on the respective domains $\Omega_{i}$ with a homogeneous Neumann BC on the parts $\Gamma_{i, \sigma}, \sigma \in \mathcal{N}_{i}$, of the boundary $\partial \Omega_{i}, 1 \leq i \leq M$. The stiffness matrices have to be modified and assembled in a suitable way so as to take properly into account the TC (1.2) in the nodes of each interface $\Gamma_{i, \sigma}$, to be counted once as a node of $\bar{\Omega}_{i}$ and once as a node of $\bar{\Omega}_{\sigma}$.

\section{Numerical QUADRATURE FINITE ELEMENT METHOD}

5.1. Preliminaries (cf. [3], [6] and [14, §2.1]). Consider the (affine invertible) mapping

$$
F_{K}: \widehat{K} \text { (reference element) } \rightarrow K: \widehat{x} \mapsto x=F_{K}(\widehat{x})=B_{K} \widehat{x}+b_{K}, \operatorname{det} B_{K}>0,
$$

with $B_{K} \in \mathbb{R}^{2 \times 2}$ and $b_{K} \in \mathbb{R}^{2 \times 1}, K \in \mathcal{T}_{h_{i}}^{i}$.

Next, introduce a quadrature formula on $\widehat{K}$,

$$
I_{\widehat{K}}^{X}(\widehat{\varphi})=\sum_{r=1}^{N(X)} \widehat{\omega}_{r}^{X} \widehat{\varphi}\left(\widehat{b}_{r}^{X}\right) \simeq \int_{\widehat{K}} \widehat{\varphi}(\widehat{x}) \mathrm{d} \widehat{x}, \quad \forall \widehat{\varphi} \in \mathcal{C}^{0}(\widehat{K}), X=L, G,
$$

where $\widehat{b}_{r}^{X}$ and $\widehat{\omega}_{r}^{X}>0, r=1, \ldots, N(X)$, are the quadrature nodes and weights, respectively, and where $X=L$ and $X=G$ refer to the quadrature formulas having degree of precision $2 k-1$ and $2 k+1$, respectively. The quadrature error is

$$
E_{\widehat{K}}^{X}(\widehat{\varphi}) \equiv \int_{\widehat{K}} \widehat{\varphi}(\widehat{x}) \mathrm{d} \widehat{x}-I_{\widehat{K}}^{X}(\widehat{\varphi})
$$


Putting $\varphi(x)=\widehat{\varphi}(\widehat{x})$ whenever $x=F_{K}(\widehat{x}), \widehat{x} \in \widehat{K}$, we define the corresponding quadrature formulas on $K$ by

$$
I_{K}^{X}(\varphi)=\left(\operatorname{det} B_{K}\right) I_{\widehat{K}}^{X}(\widehat{\varphi}) \simeq \int_{K} \varphi(x) \mathrm{d} x, \quad X=L, G .
$$

In a similar way we approximate the line integrals on a side $\partial_{t} \widehat{K}$ of $\widehat{K}$, using a one-dimensional Lobatto $(X=L)$ or Gauss-Legendre $(X=G)$ quadrature formula

$$
I_{\partial_{t} \widehat{K}}^{X}(\widehat{\varphi})=\sum_{r=1}^{k+1} \widehat{\omega}_{r}^{X} \widehat{\varphi}\left(\widehat{g}_{r}^{X}\right) \simeq \int_{\partial_{t} \widehat{K}} \widehat{\varphi}(\widehat{s}) \mathrm{d} \widehat{s}, \quad \forall \widehat{\varphi} \in \mathcal{C}^{0}\left(\partial_{t} \widehat{K}\right),
$$

where the quadrature points $\widehat{g}_{r}^{X}$ (characterized by their arc length) correspond to the Lobatto or Gauss points in the interval $[-1,1]$, respectively. This quadrature formula has precision $2 k-1$ or $2 k+1$, respectively; see [6, §2.7].

According to (5.1) we put $\varphi(s)=\widehat{\varphi}(\widehat{s}), s=\left.F_{K}\right|_{\partial_{t} \widehat{K}}(\widehat{s}), \widehat{s} \in \partial_{t} \widehat{K}$. The quadrature formula on a side $\partial_{t} K$ of an element $K$ is defined by

$$
I_{\partial_{t} K}^{X}(\varphi)=\frac{\text { meas } \partial_{t} K}{\operatorname{meas} \partial_{t} \widehat{K}} I_{\partial_{t} \widehat{K}}^{X}(\widehat{\varphi}) .
$$

We refer to [6, pp. 398-399], [8, pp. 2.122-2.124] and to [3, pp. 181-184], [5, §XII 1.5, p. 780], [8, pp. 2.100-2.104] for examples of quadrature formulas on a rectangular and a triangular element $K$, respectively.

5.2. Approximation of the inner product of $H$. We define the discrete inner product of $H$ and the associated norm in $X_{h}$ by

$$
(v, w)_{h}=\sum_{i=1}^{M} \sum_{K \in \mathcal{T}_{h_{i}}^{i}} I_{K}^{L}\left(v^{i} w^{i}\right), \quad|v|_{h}=\sqrt{(v, v)_{h}}, \quad \forall v, w \in X_{h} .
$$

Proposition 5.1. The norms $|\cdot|_{h},(5.4)$, and $|\cdot|,(2.1)$, are uniformly equivalent on the space $X_{h}$, i.e., there exist constants $c_{1}, c_{2}>0$ independent of $h$ such that

$$
c_{1}|v| \leq|v|_{h} \leq c_{2}|v|, \quad \forall v \in X_{h} .
$$

Proof. Apply [1, Lemma 3.2] or the analogue for a triangular mesh on a onecomponent domain.

5.3. Approximation of the bilinear form. First, introducing the notations

$\mathcal{N}\left(\Gamma_{2}^{i}\right)=\left\{j: 1 \leq j \leq N\left(\Omega_{i}\right)\right.$ and $\partial_{j} \Omega_{i}$ (side of $\left.\left.\Omega_{i}\right) \subset \Gamma_{2}^{i}\right\}$,

$$
\begin{aligned}
\mathcal{B}_{j, h_{i}} & =\left\{K \in \mathcal{T}_{h_{i}}^{i}: \exists t: \partial_{t} K \subset \partial_{j} \Omega_{i}\right\}, j \in \mathcal{N}\left(\Gamma_{2}^{i}\right), \\
\mathcal{B}_{\sigma, h_{i}}^{i} & =\left\{K \in \mathcal{T}_{h_{i}}^{i}: \exists t: \partial_{t} K \subset \Gamma_{i, \sigma}\right\}, \sigma \in \mathcal{N}_{i},
\end{aligned}
$$

$\left(N\left(\Omega_{i}\right)\right.$ number of sides of $\left.\Omega_{i}\right)$

with $t \in\{1,2,3,4\}$ when $K$ is rectangular and $t \in\{1,2,3\}$ when $K$ is triangular, we have

$$
\begin{aligned}
\int_{\Gamma_{2}^{i}} \varphi(s) \mathrm{d} s & =\sum_{j \in \mathcal{N}\left(\Gamma_{2}^{i}\right)} \sum_{K \in \mathcal{B}_{j, h_{i}}} \int_{\partial_{t} K} \varphi(s) \mathrm{d} s, \quad \forall \varphi \in \mathcal{C}^{0}\left(\overline{\Gamma_{2}^{i}}\right), \\
\int_{\Gamma_{i, \sigma}} \varphi(s) \mathrm{d} s & =\sum_{K \in \mathcal{B}_{\sigma, h_{i}}^{i}} \int_{\partial_{t} K} \varphi(s) \mathrm{d} s, \quad \forall \varphi \in \mathcal{C}^{0}\left(\bar{\Gamma}_{i, \sigma}\right) .
\end{aligned}
$$


In what follows, we assume that $a_{\ell m}^{i}, a_{0}^{i} \in \mathcal{C}^{0}\left(\bar{\Omega}_{i}\right), \ell, m=1,2, h^{i, \sigma} \in \mathcal{C}^{0}\left(\bar{\Gamma}_{i, \sigma}\right)$, $\sigma \in \mathcal{N}_{i}$ and that $a_{1}^{i} \in \mathcal{C}^{0}\left(\overline{\Gamma_{2}^{i}}\right), 1 \leq i \leq M$. The discrete analogue of the bilinear form $a(\cdot, \cdot),(2.5)$, on $X_{h} \times X_{h}$ is defined by

$$
a_{h}(v, w)=\mathcal{A}_{h}(v, w)+\mathcal{B}_{h}(v, w)+\mathcal{C}_{h}(v, w), \quad \forall v, w \in X_{h},
$$

where

$$
\begin{aligned}
\mathcal{A}_{h}(v, w) & =\sum_{i=1}^{M} \mathcal{A}_{h_{i}}^{i}\left(v^{i}, w^{i}\right) \\
& =\sum_{i=1}^{M} \sum_{K \in \mathcal{T}_{h_{i}}^{i}} I_{K}^{X}\left(\sum_{\ell, m=1}^{2} a_{\ell m}^{i} \partial_{\ell} v^{i} \partial_{m} w^{i}+a_{0}^{i} v^{i} w^{i}\right) \\
\mathcal{B}_{h}(v, w) & =\sum_{i=1}^{M} \mathcal{B}_{h_{i}}^{i}\left(v^{i}, w^{i}\right) \\
& =\sum_{i=1}^{M} \frac{1}{2} \sum_{\sigma \in \mathcal{N}_{i}} \sum_{K \in \mathcal{B}_{\sigma, h_{i}}^{i}} I_{\partial_{t} K}^{X}\left(h^{i, \sigma}\left(v^{i}-v^{\sigma}\right)\left(w^{i}-w^{\sigma}\right)\right), \\
\mathcal{C}_{h}(v, w) & =\sum_{i \in \mathcal{N}\left(\Gamma_{2}\right)} \mathcal{C}_{h_{i}}^{i}\left(v^{i}, w^{i}\right)=\sum_{i \in \mathcal{N}\left(\Gamma_{2}\right)} \sum_{j \in \mathcal{N}\left(\Gamma_{2}^{i}\right)} \sum_{K \in \mathcal{B}_{j, h_{i}}} I_{\partial_{t} K}^{X}\left(a_{1}^{i} v^{i} w^{i}\right) .
\end{aligned}
$$

Obviously, $a_{h}(\cdot, \cdot)$ retains the symmetry property of $a(\cdot, \cdot)$. Further, we obtain:

Proposition 5.2. The form $a_{h}(\cdot, \cdot),(5.5)$, is uniformly bounded and strongly coercive, i.e.,

$$
\begin{aligned}
& \text { (1) } \exists c_{3}>0:\left|a_{h}(v, w)\right| \leq c_{3}\|v\|\|w\|, \quad \forall v, w \in X_{h}, \forall h . \\
& \text { (2) } \exists c_{4}>0: \quad a_{h}(v, v) \geq c_{4}\|v\|^{2}, \quad \forall v \in V_{h}, \forall h \text {. }
\end{aligned}
$$

Proof. (1) Proceeding along similar lines as in [3, p. 187], we find (cf. [14, (2.12)])

$$
\left|\mathcal{A}_{h_{i}}^{i}\left(v^{i}, w^{i}\right)+\mathcal{C}_{h_{i}}^{i}\left(v^{i}, w^{i}\right)\right| \leq c_{0}^{i}\left\|v^{i}\right\|_{1, \Omega_{i}}\left\|w^{i}\right\|_{1, \Omega_{i}}, \quad \forall v, w \in X_{h}, 1 \leq i \leq M,
$$

where $c_{0}^{i}$ is independent of $h_{i}$. Hence,

$$
\left|\mathcal{A}_{h}(v, w)+\mathcal{C}_{h}(v, w)\right| \leq C\|v\|\|w\|, \quad \forall v, w \in X_{h} .
$$

For $X=G$ the precision of the quadrature formula leads to

$$
\left|\mathcal{B}_{h}(v, w)\right| \leq \frac{1}{2} \sum_{i=1}^{M} \sum_{\sigma \in \mathcal{N}_{i}}\left|h^{i, \sigma}\right|_{0, \infty, \Gamma_{i, \sigma}} \sum_{j, \rho \in\{i, \sigma\}} \int_{\Gamma_{i, \sigma}}\left|v^{j} w^{\rho}\right| \mathrm{d} s .
$$

For $X=L$ this estimate is seen to hold also by first showing, as in [1, Lemma 3.2], that

$$
\exists C>0: I_{\partial_{t} K}^{L}\left(p^{2}\right) \leq C \int_{\partial_{t} K} p^{2} \mathrm{~d} s, \quad \forall p \in P_{k}\left(\partial_{t} K\right), \forall h .
$$

Hence, from the trace inequality,

$$
\left|\mathcal{B}_{h}(v, w)\right| \leq C \sum_{i=1}^{M} \sum_{\sigma \in \mathcal{N}_{i}} \sum_{j, \rho \in\{i, \sigma\}}\left\|v^{j}\right\|_{1, \Omega_{j}}\left\|w^{\rho}\right\|_{1, \Omega_{\rho}} \leq C\|v\|\|w\|, \quad \forall v, w \in X_{h} .
$$


(2) To prove (5.10), note that $[15,(2.8)]$, or the analogous result for a triangular mesh, leads to

$$
\mathcal{A}_{h}(v, v) \geq \sum_{i=1}^{M} c^{i}\left\|v^{i}\right\|_{1, \Omega_{i}}^{2} \geq \min _{1 \leq i \leq M} c^{i}\|v\|^{2}, \quad \forall v \in V_{h},
$$

where $c^{i}>0$ are constants independent of $h_{i}, 1 \leq i \leq M$. Moreover, use (H1)(2)(3).

5.4. Estimate of the total error of quadrature. The approximate inner product (5.4) and bilinear form (5.5) involve the respective errors

$$
\begin{aligned}
E^{L}(v, w) & \equiv(v, w)-(v, w)_{h}=\sum_{i=1}^{M} \sum_{K \in \mathcal{T}_{h_{i}}^{i}} E_{K}^{L}\left(v^{i} w^{i}\right), \quad \forall v, w \in X_{h}, \\
E_{a}^{X}(v, w) & \equiv a(v, w)-a_{h}(v, w) \\
& =E_{\mathcal{A}}^{X}(v, w)+E_{\mathcal{B}}^{X}(v, w)+E_{\mathcal{C}}^{X}(v, w), \quad \forall v, w \in X_{h},
\end{aligned}
$$

where $E_{\mathcal{A}}^{X}, E_{\mathcal{B}}^{X}$ and $E_{\mathcal{C}}^{X}$ are given by expressions similar to (5.6), (5.7) and (5.8), respectively, in terms of $E_{K}^{X}$ and $E_{\partial_{t} K}^{X}$, the errors of quadrature on an element $K$ and on a side of $K$.

These last (local) errors are estimated in the following three propositions, the proofs of which are similar to those of [1, Theorem 3.5-Corollary 3.6], [2, Lemmas 3.1-3.2] and [14, Theorems 2.5-2.6-2.7]. In these estimates $C$ is a generic constant not depending on $K$ while $h_{K}$ stands for the diameter of $K$.

Proposition 5.3. For the quadrature formula $(5.2)(X=L)$ we have

$$
\left|E_{K}^{L}(p q)\right| \leq C h^{2 k-s-t}|p|_{k-s, K}|q|_{k-t, K}, \quad \forall p, q \in P(K), 0 \leq s, t \leq k .
$$

Proposition 5.4. For the quadrature formula (5.2) we have, for $0 \leq r \leq k$,

$$
\begin{gathered}
\left|E_{K}^{L}(d p q)\right| \leq C h_{K}^{r}\|d\|_{r, \infty, K}\|p\|_{r, K}|q|_{0, K}, \quad \forall d \in W^{r, \infty}(K), \forall p, q \in P_{k}(K), \\
\left|E_{K}^{G}(d p q)\right| \leq C h_{K}^{r+2}\|d\|_{r+2, \infty, K}\|p\|_{\min (r+2, k), K}|q|_{0, K}, \\
\forall d \in W^{r+2, \infty}(K), \forall p, q \in P(K) .
\end{gathered}
$$

For $k=1$ we also have

$$
\left|E_{K}^{G}(d p q)\right| \leq C h_{K}\|d\|_{1, \infty, K}\|p\|_{1, K}|q|_{0, K}, \quad \forall d \in W^{1, \infty}(K), \forall p, q \in P(K) .
$$

When $d \in W^{2 k, \infty}(K)$ we have

$$
\left|E_{K}^{L}(d p q)\right| \leq C h_{K}^{2 k}\|d\|_{2 k, \infty, K}\|p\|_{k, K}\|q\|_{k, K}, \quad \forall p, q \in P_{k}(K) .
$$

Proposition 5.5. For the quadrature formula (5.3) we have the same estimates as (5.14)-(5.17), evidently with $K$ replaced by $\partial_{t} K$.

These estimates for the local errors lead to the estimates of the total quadrature errors (5.11) and (5.12).

Theorem 5.1. For the total error of quadrature $E^{L}(\cdot, \cdot),(5.11)$, we have

$$
\left|E^{L}(v, w)\right| \leq C h^{2}|v|_{\widehat{H}^{1}(\Omega)}|w|_{\widehat{H}^{1}(\Omega)}, \quad \forall v, w \in X_{h} .
$$

Proof. Apply (5.13) with $s=t=k-1$ and proceed similarly as in [1, Lemma 5.1]. 
Theorem 5.2. For the total errors of quadrature $E_{a}^{X}(\cdot, \cdot),(5.12)$, we have:

(1) Let $a_{\ell m}^{i} \in W^{k+s, \infty}\left(\Omega_{i}\right), \ell, m=1,2, a_{0}^{i} \in W^{k, \infty}\left(\Omega_{i}\right)$ and $h^{i, \sigma} \in W^{k+s, \infty}\left(\Gamma_{i, \sigma}\right)$, $\sigma \in \mathcal{N}_{i}, 1 \leq i \leq M$. Further, let $a_{1}^{i} \in W^{k+s, \infty}\left(\Gamma_{2}^{i}\right), i \in \mathcal{N}\left(\Gamma_{2}\right)$. Here, $s=1$ when dealing with a rectangular mesh and $s=0$ for a triangular mesh. Then

$$
\left|E_{a}^{G}(v, w)\right| \leq C h\|v\|\|w\|, \quad \forall v, w \in X_{h} .
$$

(2) When the order of the Sobolev spaces involved in all the regularity conditions in (1) is increased by one unit, then

$$
\left|E_{a}^{G}(v, w)\right| \leq C h^{2}\|v\|\|w\|, \quad \forall v, w \in X_{h} .
$$

(3) Let $a_{\ell m}^{i}, a_{0}^{i} \in W^{\alpha k, \infty}\left(\Omega_{i}\right), \ell, m=1,2, h^{i, \sigma} \in W^{\alpha k, \infty}\left(\Gamma_{i, \sigma}\right), \sigma \in \mathcal{N}_{i}, 1 \leq$ $i \leq M$, and let $a_{1}^{i} \in W^{\alpha k, \infty}\left(\Gamma_{2}^{i}\right), i \in \mathcal{N}\left(\Gamma_{2}\right), \alpha=1,2$. Then, for the case of a triangular mesh, we have

$$
\left|E_{a}^{L}(v, w)\right| \leq C h^{\alpha}\|v\|\|w\|, \quad \forall v, w \in X_{h} .
$$

Proof. (1) Apply (5.15) with $r=k-2+s$, combined with (5.16) when $s=0$, to the components constituting $E_{\mathcal{A}}^{G}$. Use the analogous estimates for the terms constituting $E_{\mathcal{C}}^{G}$ and invoke the trace inequality as well as (3.2) (possibly combined with Remark 3.1). We get

$$
\left|E_{\mathcal{A}}^{G}(v, w)+E_{\mathcal{C}}^{G}(v, w)\right| \leq C h\|v\|\|w\|, \quad \forall v, w \in X_{h} .
$$

Recalling that $h^{i, \sigma}=h^{\sigma, i}$ and using arguments similar to those for $E_{\mathcal{C}}^{G}$, we find

$$
\begin{aligned}
&\left|E_{\mathcal{B}}^{G}(v, w)\right| \leq C \sum_{i=1}^{M} h_{i}^{k+s} \sum_{\sigma \in \mathcal{N}_{i}}\left\|h^{i, \sigma}\right\|_{k+s, \infty, \Gamma_{i, \sigma}} \sum_{K \in \mathcal{B}_{\sigma, h_{i}}^{i}}\left\|w^{i}\right\|_{k, \partial_{t} K}\left(\left|v^{i}\right|_{0, \partial_{t} K}+\left|v^{\sigma}\right|_{0, \partial_{t} K}\right) \\
& \leq C \sum_{i=1}^{M} h_{i} \sum_{\sigma \in \mathcal{N}_{i}} \sum_{K \in \mathcal{B}_{\sigma, h_{i}}^{i}}\left\|w^{i}\right\|_{1, K}\left(\left\|v^{i}\right\|_{1, K}+\left|v^{\sigma}\right|_{0, \partial_{t} K}\right) \\
& \leq C h \sum_{i=1}^{M} \sum_{\sigma \in \mathcal{N}_{i}}\left\|w^{i}\right\|_{1, \Omega_{i}}\left(\left\|v^{i}\right\|_{1, \Omega_{i}}+\left\|v^{\sigma}\right\|_{1, \Omega_{\sigma}}\right) \leq C h\|w\|\|v\|, \\
& \forall v, w \in X_{h},
\end{aligned}
$$

where in the penultimate step we noted that

$$
\sum_{K \in \mathcal{B}_{\sigma, h_{i}}^{i}}\left|v^{\sigma}\right|_{0, \partial_{t} K}^{2}=\sum_{K^{\prime} \in \mathcal{B}_{i, h_{\sigma}}^{\sigma}}\left|v^{\sigma}\right|_{0, \partial_{t^{\prime}} K^{\prime}}^{2}
$$

(2) Proceed in a similar way, now taking $r=k-1+s$ in (5.15) and its analogue for $E_{\partial_{t} K}^{G}$.

(3) When $\alpha=1$ use (5.14) and its analogue for $E_{\partial_{t} K}^{L}$ with $r=k$. When $\alpha=2$ invoke (5.17) and its analogue.

Remark 5.1. The estimates (5.18), (5.19)-(5.20) and (5.21) for $E^{L}, E_{a}^{G}$ and $E_{a}^{L}$, respectively, are formally the same as those for an EVP in a one-component domain. 
5.5. Approximate eigenvalue problem with numerical quadrature. The approximate eigenvalue problem with numerical quadrature reads:

$$
\text { Find }\left(\widehat{\lambda}_{h}, \widehat{u}_{h}\right) \in \mathbb{R} \times V_{h}: a_{h}\left(\widehat{u}_{h}, v\right)=\widehat{\lambda}_{h}\left(\widehat{u}_{h}, v\right)_{h}, \quad \forall v \in V_{h} .
$$

The properties of $a_{h}(\cdot, \cdot)$ and $(\cdot, \cdot)_{h}$ mentioned in Propositions 5.1 and 5.2 (combined with the symmetry of $\left.a_{h}(\cdot, \cdot)\right)$ guarantee the existence of approximate eigenpairs, denoted by $\left(\widehat{\lambda}_{\ell, h}, \widehat{u}_{\ell, h}\right), 1 \leq \ell \leq N(h)$; all eigenvalues are strictly positive and the eigenfunctions are orthogonal with respect to $(\cdot, \cdot)_{h}$.

Remark 5.2. For the stiffness matrix and the mass matrix of the algebraic EVP corresponding to (5.22) a similar remark applies as for the consistent mass FEM.

5.6. Convergence and error estimate of the approximate eigenpairs. The approach outlined so far, starting from the variational formulation (2.6) of the original EVP, through its numerical quadrature FE approximation, is formally identical to the treatment of a class of elliptic EVPs in one-component domains, considered in [14]. In particular, the key results, obtained in the previous section, on the basis of Propositions 5.1-5.2, are, mutatis mutandis, similar to those in [14, §3-4], even in the presence of the transmission part $\mathcal{B}(\cdot, \cdot)$ entering the bilinear form $a(\cdot, \cdot)$ on account of the jump conditions at the interfaces of the subregions. Therefore, the error estimates for the numerical quadrature FE approximate eigenpairs will have the same structure as those in the paper just cited, requiring of course suitable interpretations of all quantities and conditions involved, according to the setting of $\S 2.1$ and $\S 3.1$.

Let $\lambda_{\ell}$ be an $(L+1)$-fold eigenvalue of (2.6) $(0 \leq L<+\infty)$. By $u_{\ell}, \ldots, u_{\ell+L}$ we denote a set of associated eigenfunctions. Let $\left(\widehat{\lambda}_{\ell+r, h}, \widehat{u}_{\ell+r, h}\right), r=0, \ldots, L$, be the corresponding eigenpairs of (5.22). The exact eigenfunctions may be taken to be orthonormal in $H$, while the approximate eigenfunctions may be taken to be orthonormal with respect to $(\cdot, \cdot)_{h}$.

Theorem 5.3. (1) Assume that the coefficients $a_{\ell m}^{i}, a_{0}^{i}$, $a_{1}^{i}$ and $h^{i, \sigma}$ satisfy the conditions underlying the estimate (5.19). Then, we have for both $X=L$ and $X=G$

$$
\widehat{\lambda}_{\ell+r, h} \rightarrow \lambda_{\ell} \quad \text { as } \quad h \rightarrow 0, \quad r=0, \ldots, L .
$$

(2) Assume that the coefficients $a_{\ell m}^{i}, a_{0}^{i}, a_{1}^{i}$ and $h^{i, \sigma}$ satisfy the regularity conditions underlying the estimate (5.20) or (5.21) (with $r=2$ ) [when using $X=L$ for a triangular mesh]. Moreover, assume that the exact eigenfunctions $u_{1}, \ldots, u_{\ell+L}$ belong to $\widehat{H}^{k+1}(\Omega)$. Then, we have for both $X=L$ and $X=G$

$$
\left|\widehat{\lambda}_{\ell+r, h}-\lambda_{\ell}\right| \leq C(\ell) h^{2}, \quad r=0, \ldots, L .
$$

Case of a simple exact eigenvalue $\lambda_{\ell}$ and $X=G$.

Theorem 5.4. (1) Let $a_{\ell m}^{i} \in W^{k+1, \infty}\left(\Omega_{i}\right), \ell, m=1,2, a_{0}^{i} \in W^{k, \infty}\left(\Omega_{i}\right)$ and $h^{i, \sigma} \in$ $W^{k+1, \infty}\left(\Gamma_{i, \sigma}\right), \sigma \in \mathcal{N}_{i}, 1 \leq i \leq M$. Further, let $a_{1}^{i} \in W^{k+1, \infty}\left(\Gamma_{2}^{i}\right), i \in \mathcal{N}\left(\Gamma_{2}\right)$. Assume that $u_{\ell} \in \widehat{H}^{k+1}(\Omega)$. Then, for $k \geq 2$, we have

$$
\left|u_{\ell}-\widehat{u}_{\ell, h}\right| \leq C(\ell) h^{k-1} \text {. }
$$


(2) Let $a_{\ell m}^{i}, a_{0}^{i} \in W^{2 k, \infty}\left(\Omega_{i}\right), \ell, m=1,2, h^{i, \sigma} \in W^{2 k, \infty}\left(\Gamma_{i, \sigma}\right), \sigma \in \mathcal{N}_{i}, 1 \leq i \leq$ $M$, and let $a_{1}^{i} \in W^{2 k, \infty}\left(\Gamma_{2}^{i}\right), i \in \mathcal{N}\left(\Gamma_{2}\right)$. Assume that $u_{\ell} \in \widehat{H}^{k+1}(\Omega)$. Then, for $k \geq 2$, we have

$$
\begin{aligned}
\left|\lambda_{\ell}-\widehat{\lambda}_{\ell, h}\right| & \leq C(\ell) h^{2 k-1}, \\
\left\|u_{\ell}-\widehat{u}_{\ell, h}\right\| & \leq C(\ell) h^{k-1} .
\end{aligned}
$$

Case of a multiple exact eigenvalue $\lambda_{\ell}$ and $X=G$.

Theorem 5.5. (1) Retain the same conditions on the data as in Theorem5.4(1). Assume that $u_{\ell}, \ldots, u_{\ell+L} \in \widehat{H}^{k+1}(\Omega)$. Then, for $k \geq 2$, there exist a set $\left(W_{\ell+r}\right)_{r=0}^{L}$ of fixed exact eigenfunctions corresponding to the $(L+1)$-fold exact eigenvalue $\lambda_{\ell}$ and being orthonormal in $H$, a sequence $\left\{h_{j}\right\}$ with $h_{j} \rightarrow 0$ and a number $m$, $0<m \leq k-1$, such that

$$
\left|W_{\ell+r}-\widehat{u}_{\ell+r, h_{j}}\right| \leq C(\ell) h_{j}^{m}, \quad r=0, \ldots, L .
$$

(2) Retain the same conditions on the data as in Theorem 5.4(2). Assume that $u_{\ell}, \ldots, u_{\ell+L} \in \widehat{H}^{k+1}(\Omega)$. Then, for $k \geq 2$, we have

$$
\left|\lambda_{\ell}-\widehat{\lambda}_{\ell+r, h}\right| \leq C(\ell) h^{2 k-1}, \quad r=0, \ldots, L .
$$

(3) Under the same assumptions as in (2), there exist a set $\left(W_{\ell+r}\right)_{r=0}^{L}$ of fixed exact eigenfunctions corresponding to the $(L+1)$-fold exact eigenvalue $\lambda_{\ell}$ and being orthonormal in $H$, a sequence $\left\{h_{j}\right\}$ with $h_{j} \rightarrow 0$ and a number $m, 0<m \leq k-1$, such that

$$
\left\|W_{\ell+r}-\widehat{u}_{\ell+r, h_{j}}\right\| \leq C(\ell) h_{j}^{m}, \quad r=0, \ldots, L .
$$

Less useful estimates may be obtained for $m=k-1$ and for all $h$, for a set $\left(U_{\ell+r, \star}(h)\right)_{r=0}^{L}$ of exact eigenfunctions depending, however, on $h$.

Remark 5.4. In Theorems 5.4-5.5 the rates of convergence are only suboptimal. This suboptimality can be regarded as a limitation of the purely variational method which we have outlined. In fact, by extending the arguments from [14], we can improve these rates of convergence by one unit, but at the cost of a supplementary assumption, viz.

the boundary value problem associated with the bilinear form $a(\cdot, \cdot)$, (2.5), is 'regular' in the sense of [3, p. 138], i.e., the solution operator $T: H \rightarrow V$ defined by

$$
a(T f, v)=(f, v), \quad \forall f \in H, \forall v \in V,
$$

is a bounded linear operator from $H$ into $\widehat{H}^{2}(\Omega) \equiv H^{2}\left(\Omega_{1}\right) \times \cdots \times$ $H^{2}\left(\Omega_{M}\right)$.

This type of assumption is well known in finite element error analysis, but for the present EVP no set of sufficient conditions for the data of (1.1)-(1.5) can be given guaranteeing the validity of this assumption.

Case of a triangular mesh and $X=L$.

When using a less accurate numerical quadrature formula to approximate the bilinear form, viz. when we take $X=L$ in (5.5)-(5.8), the same rate of convergence for the approximate eigenpairs may be recovered. However, for the error estimate of the eigenfunctions in the $H$-norm this requires the same regularity conditions as in Theorem 5.4(2). 


\section{NumericAl EXAMPLE}

The effectiveness of the variational formulation of the multi-component EVP and its (consistent mass and numerical quadrature) FE approximation is tested for a 1D model problem, the eigenpairs of which can be evaluated exactly (at least in principle).

TABLE 6.1

\begin{tabular}{|c|c|c|c|c|c|}
\hline & & & & \multicolumn{1}{|c|}{ Relative error in \% } \\
\hline N1 & N2 & CM FEM & LM FEM & CM & LM \\
\hline \hline \multicolumn{6}{|c|}{ Eigenvalue 1: 'exact' eigenvalue is 0.6099903 } \\
\hline 40 & 40 & 0.6100017 & 0.6099685 & $1.87 \mathrm{E}-3$ & $-3.57 \mathrm{E}-3$ \\
80 & 80 & 0.6099932 & 0.6099849 & $4.68 \mathrm{E}-4$ & $-8.93 \mathrm{E}-4$ \\
160 & 160 & 0.6099910 & 0.6099890 & $1.17 \mathrm{E}-4$ & $-2.23 \mathrm{E}-4$ \\
320 & 320 & 0.6099905 & 0.6099900 & $2.92 \mathrm{E}-5$ & $-5.57 \mathrm{E}-5$ \\
640 & 640 & 0.6099904 & 0.6099902 & $7.29 \mathrm{E}-6$ & $-1.38 \mathrm{E}-5$ \\
1280 & 1280 & 0.6099903 & 0.6099903 & $1.88 \mathrm{E}-6$ & $-3.31 \mathrm{E}-6$ \\
\hline \multicolumn{6}{|c|}{ Eigenvalue 2: 'exact' eigenvalue is 6.489800 } \\
\hline 40 & 40 & 6.491761 & 6.488773 & $3.02 \mathrm{E}-2$ & $-1.58 \mathrm{E}-2$ \\
80 & 80 & 6.490290 & 6.489543 & $7.56 \mathrm{E}-3$ & $-3.96 \mathrm{E}-3$ \\
160 & 160 & 6.489922 & 6.489736 & $1.89 \mathrm{E}-3$ & $-9.89 \mathrm{E}-4$ \\
320 & 320 & 6.489830 & 6.489784 & $4.72 \mathrm{E}-4$ & $-2.47 \mathrm{E}-4$ \\
640 & 640 & 6.489807 & 6.489796 & $1.18 \mathrm{E}-4$ & $-6.18 \mathrm{E}-4$ \\
1280 & 1280 & 6.489802 & 6.489799 & $2.95 \mathrm{E}-5$ & $-1.54 \mathrm{E}-5$ \\
\hline \multicolumn{6}{|c|}{ Eigenvalue 3: 'exact' eigenvalue is 20.88131 } \\
\hline 40 & 40 & 20.89752 & 20.86457 & $7.76 \mathrm{E}-2$ & $-8.02 \mathrm{E}-2$ \\
80 & 80 & 20.88537 & 20.87713 & $1.94 \mathrm{E}-2$ & $-2.00 \mathrm{E}-2$ \\
160 & 160 & 20.88233 & 20.88027 & $4.85 \mathrm{E}-3$ & $-5.01 \mathrm{E}-3$ \\
320 & 320 & 20.88157 & 20.88105 & $1.21 \mathrm{E}-3$ & $-1.25 \mathrm{E}-3$ \\
640 & 640 & 20.88138 & 20.88125 & $3.03 \mathrm{E}-4$ & $-3.13 \mathrm{E}-4$ \\
1280 & 1280 & 20.88133 & 20.88130 & $7.58 \mathrm{E}-5$ & $-7.83 \mathrm{E}-5$ \\
\hline \multicolumn{6}{|c|}{ Eigenvalue 4: 'exact' eigenvalue is 32.93464 } \\
\hline 40 & 40 & 32.98054 & 32.90339 & $1.39 \mathrm{E}-1$ & $-9.49 \mathrm{E}-2$ \\
80 & 80 & 32.94611 & 32.92683 & $3.48 \mathrm{E}-2$ & $-2.37 \mathrm{E}-2$ \\
160 & 160 & 32.93751 & 32.93269 & $8.70 \mathrm{E}-3$ & $-5.93 \mathrm{E}-3$ \\
320 & 320 & 32.93536 & 32.93415 & $2.18 \mathrm{E}-3$ & $-1.48 \mathrm{E}-3$ \\
640 & 640 & 32.93482 & 32.93452 & $5.44 \mathrm{E}-4$ & $-3.71 \mathrm{E}-4$ \\
1280 & 1280 & 32.93469 & 32.93461 & $1.36 \mathrm{E}-4$ & $-9.26 \mathrm{E}-5$ \\
\hline
\end{tabular}

For a domain with two components $(0,1)$ and $(1,2)$, we consider the following EVP:

Find $\lambda \in \mathbb{R}$ and the functions $u^{1}:[0,1] \rightarrow \mathbb{R}$ and $u^{2}:[1,2] \rightarrow \mathbb{R}$ obeying

the differential equations

$$
\begin{aligned}
& -\left(u^{1}\right)^{\prime \prime}=\lambda u^{1} \quad \text { in }(0,1), \\
& -2\left(u^{2}\right)^{\prime \prime}=\lambda u^{2} \quad \text { in }(1,2) \text {, }
\end{aligned}
$$


as well as the transmission conditions

$$
\begin{aligned}
-\left(u^{1}\right)^{\prime} & =5\left(u^{1}-u^{2}\right) & & \text { at } x=1, \\
\left(u^{1}\right)^{\prime} & =2\left(u^{2}\right)^{\prime} & & \text { at } x=1,
\end{aligned}
$$

and the boundary conditions

$$
u^{1}(0)=\left(u^{2}\right)^{\prime}(2)=0
$$

The eigenvalue equation for $\mu=\sqrt{\lambda}$ is easily found to be

$$
\frac{\mu}{5}+\tan \mu-\frac{1}{\sqrt{2}} \cot \frac{\mu}{\sqrt{2}}=0 .
$$

For the finite element discretization, we consider linear shape functions on a uniform mesh with $N$ elements in each of the components; hence $h=1 / N$. We compare the first four eigenvalues, obtained from (6.1), with the numerical eigenvalues, obtained with respectively a consistent mass (CM) FEM and a lumped mass (LM) FEM. The results are given in Table 6.1. They confirm the theoretical order of convergence, namely $O\left(h^{2}\right)$, see (4.4) $(r=1)$ and Theorem 5.3(2). For the first eigenvalue the results are also depicted in Figures 6.1-6.2. In Figure 6.2 we note that the relative (percent) deviation $R$ of the approximate eigenvalue to the exact one (i.e., $\left.R=\left(\lambda_{\text {app }}-\lambda_{\text {ex }}\right) / \lambda_{\text {ex }} \cdot 100 \%\right)$ obeys

$$
\log |R| \approx C+\log h^{2}=C-2 \log N \quad(C \text { constant independent of } h),
$$

in accordance with the theoretical order of convergence just mentioned.

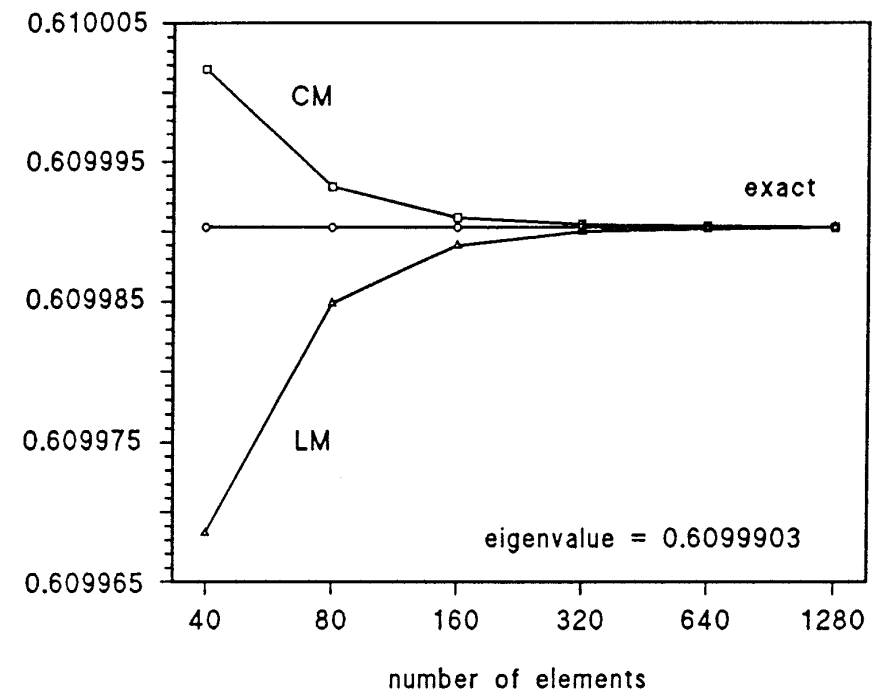

Figure 6.1. CM and LM eigenvalues 


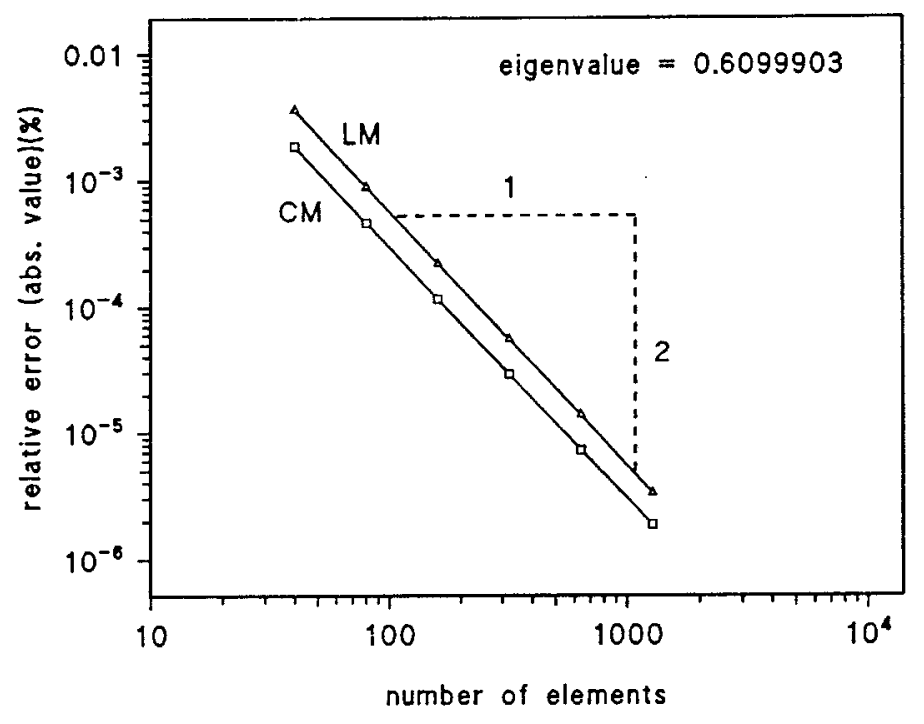

FIgURE 6.2. Rate of convergence

\section{APPENDiX: On The TCs (1.2)-(1.3)}

A simple 1D situation leading to TCs of the type (1.2)-(1.3) is shown in Figure A.1 below, cf. [12, Chapter 6]. A cavity (room) is enclosed between two walls at respective temperatures $u^{1}$ and $u^{2}$, with thermal conductivities $k^{1}$ and $k^{2}$, respectively. As usual, the walls are assumed to conduct heat in one direction only, orthogonal to the parallel surfaces, and the air in the cavity, with zero heat capacity $c_{p}$, is taken to be at temperature $T$ throughout. At the surfaces $x=x_{1}$ and $x=x_{2}$ heat is transferred by convection to the air in the cavity and by radiation (linearized here for simplicity) to the opposite wall, i.e.,

$$
\begin{gathered}
-\left.k^{1} \frac{\partial u^{1}}{\partial x}\right|_{x=x_{1}}=H_{\text {con }}^{1}\left(\left.u^{1}\right|_{x=x_{1}}-T\right)+H_{\text {rad }}^{1,2}\left(\left.u^{1}\right|_{x=x_{1}}-\left.u^{2}\right|_{x=x_{2}}\right), \\
\left.k^{2} \frac{\partial u^{2}}{\partial x}\right|_{x=x_{2}}=H_{\text {con }}^{2}\left(\left.u^{2}\right|_{x=x_{2}}-T\right)+H_{\text {rad }}^{2,1}\left(\left.u^{2}\right|_{x=x_{2}}-\left.u^{1}\right|_{x=x_{1}}\right),
\end{gathered}
$$

where $H_{\text {con }}^{1}, H_{\text {con }}^{2}$ are the convective heat transfer coefficients at the two surfaces, while $H_{\mathrm{rad}}^{1,2}=H_{\mathrm{rad}}^{2,1}$ is the coefficient of linearized radiative heat transfer between the two surfaces of the cavity (tacitly assumed to be of the same material, otherwise $\left.H_{\mathrm{rad}}^{1,2} \neq H_{\mathrm{rad}}^{2,1}\right)$. If the cavity is neither heated nor ventilated, the heat balance inside the cavity reads

$$
H_{\text {con }}^{1}\left(\left.u^{1}\right|_{x=x_{1}}-T\right)+H_{\text {con }}^{2}\left(\left.u^{2}\right|_{x=x_{2}}-T\right)=0 .
$$

'Eliminating' the cavity, i.e., substituting in (A.1)-(A.2) the expression for $T$ obtained from (A.3), we get

$$
-\left.k^{1} \frac{\partial u^{1}}{\partial x}\right|_{x=x_{1}}=h^{1,2}\left(\left.u^{1}\right|_{x=x_{1}}-\left.u^{2}\right|_{x=x_{2}}\right)=-\left.k^{2} \frac{\partial u^{2}}{\partial x}\right|_{x=x_{2}},
$$


where

$$
h^{1,2}=H_{\mathrm{rad}}^{1,2}+\frac{H_{\mathrm{con}}^{1} H_{\mathrm{con}}^{2}}{H_{\mathrm{con}}^{1}+H_{\mathrm{con}}^{2}} .
$$

Then, shifting the second wall to the left so as to be adjacent to the first one, we are left with a two-component problem in $\Omega=\Omega_{1} \cup \Omega_{2} \cup \Gamma_{1,2}$, with TCs of the type $(1.2)-(1.3)$ at the interface $\Gamma_{1,2}$; see (A.4).

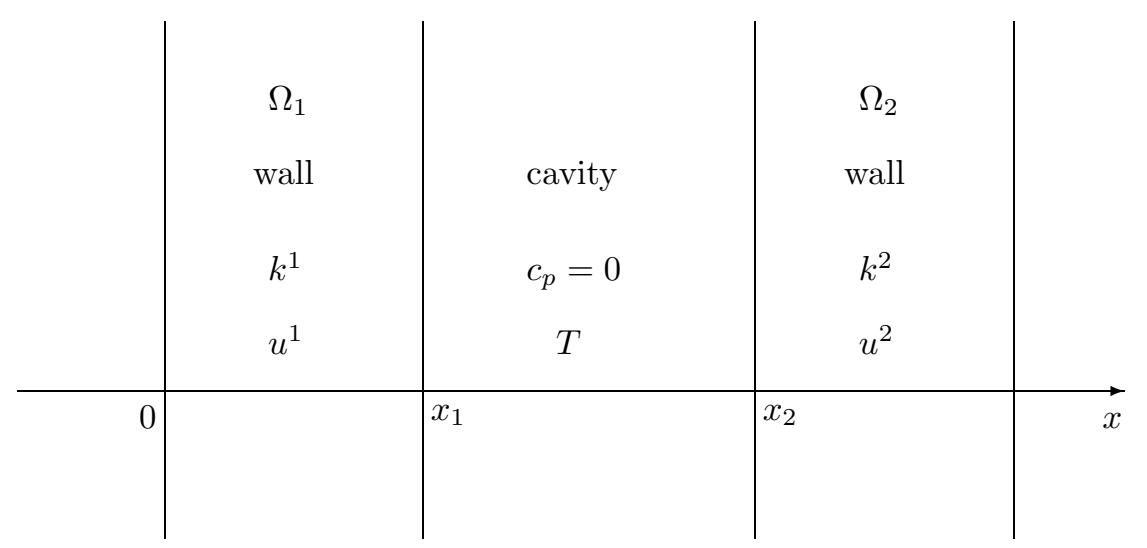

FiguRE A.1. Schematic representation of a cavity structure

\section{REFERENCES}

1. A.B. Andreev, V.A. Kascieva \& M. Vanmaele, Some results in lumped mass finite-element approximation of eigenvalue problems using numerical quadrature, J. Comp. Appl. Math., 43 (1992) 291-311. MR 93k:65088

2. U. Banerjee \& J.E. Osborn, Estimation of the Effect of Numerical Integration in Finite Element Eigenvalue Approximation, Numer. Math., 56 (1990), 735-762. MR 91d:65163

3. P.G. Ciarlet, The Finite Element Method for Elliptic Problems, North-Holland, Amsterdam (1978). MR 58:25001

4. R. Dautray \& J.-L. Lions, Analyse Mathématique et Calcul Numérique pour les Sciences et les Techniques, Tome 1, Masson, Paris (1984). MR 87g:00002

5 5_ Analyse Mathématique et Calcul Numérique pour les Sciences et les Techniques, Tome 2, Masson, Paris (1985). MR 88i:00003a

6. P.J. Davis \& P. Rabinowitz, Methods of numerical integration, Academic Press, N.Y. (1975). MR 56:7119

7. J. Kačur \& R. Van Keer, On the Numerical Solution of some Heat Transfer Problems in Multi-component Structures with nonperfect Thermal Contacts. In: R.W. Lewis, (editor), Numerical Methods for Thermal Problems VII, Pineridge Press, Swansea (1991) 1378-1388.

8. H. Kardestuncer \& D.H. Norrie, Finite Element Handbook, McGraw-Hill Book Comp., N.Y. (1987). MR 89g:65001

9. A. Kufner, O. John \& S. Fučík, Function Spaces, Noordhoff International Publishing, Leyden (1977). MR 58:2189

10. M.D. Mikhailov \& M.N. Özişik, Unified Analysis and Solutions of Heat and Mass Diffusion, John Wiley \& Sons, N.Y. (1984).

11. M.N. Özişik, Heat Conduction, (2nd edition), John Wiley \& Sons, N.Y. (1993).

12. A.W. Pratt, Heat Transmission in Buildings, J. Wiley, Chichester (1981). 
13. P.A. Raviart \& J.M. Thomas, Introduction à l'Analyse Numérique des Equations aux Dérivées Partielles, Masson, Paris (1983). MR 87a:65001a

14. M. Vanmaele, A numerical quadrature finite element method for 2nd-order eigenvalue problems with Dirichlet-Robin boundary conditions, Proceedings ISNA'92, Prague (1994), 269292.

15. M. Vanmaele \& R. Van Keer, Convergence and error estimates for a finite element method with numerical quadrature for a second-order elliptic eigenvalue problem, Int. Series of Num. Math., 96 (1991) 225-236. CMP 91:13

16. M. Vanmaele \& A. Ženíšek, External finite element approximations of eigenfunctions in the case of multiple eigenvalues, J. Comp. Appl. Math., 50 (1994), 51-66. MR 95f:65199

Department of Mathematical Analysis, Engineering Faculty, University of Gent, Galglaan 2, 9000 Gent, Belgium

E-mail address: mv@cage.rug.ac.be

E-mail address: rvk@cage.rug.ac.be 